\title{
Detection of Cognitive Impairment and Dementia Using the Animal Fluency Test: The DECIDE Study
}

\author{
Rolf Sebaldt, William Dalziel, Fadi Massoud, André Tanguay, \\ Rick Ward, Lehana Thabane, Peter Melnyk, Pierre-Alexandre Landry, \\ Benedicte Lescrauwaet
}

\begin{abstract}
Objectives: To evaluate the performance of a one-minute screening test measured against a validated 10-minute screening test for mild cognitive impairment (MCI) in detecting CI in patients aged $\geq 65$ years with two or more vascular risk factors (VRF). Methods: Patients $(n=1523)$ aged 65 years or older without documented CI symptoms or dementia with two or more VRF participated in this study set in Canadian primary care practice. Baseline data was collected, followed by the 1-minute animal fluency (AF) test and the 10-minute Montreal Cognitive Assessment (MoCA). Physicians $(n=122)$ completed case reports during patient interviews and reported their diagnostic impression. AF test sensitivity, specificity, and accuracy in predicting a positive MoCA was assessed. Results: Study sample mean age was 79.7 years, 55\% were female, $97.6 \%$ were Caucasian and $75 \%$ had $\leq 12$ years of education. The AF test and MoCA detected CI in 52 and 56 percent of the study population, respectively. The AF test demonstrated sensitivity, specificity, and accuracy in predicting a positive MoCA of 67 percent each. Physicians diagnostic impression of MCI was reported for $37 \%$ of patients, and of dementia for $6 \%$. Conclusion: In an elderly population with at least two VRF, using AF can be useful in detecting previously unknown symptoms of CI or dementia. Screening for CI in this high risk population is warranted to assist physician recognition of early CI. The short AF administration time favours its incorporation into clinical practice.
\end{abstract}

RÉSUMÉ: Détection du déficit cognitif et de la démence au moyen de l'Animal Fluency Test : l'étude DECIDE. Objectifs : Le but de l'étude était de comparer le résultat d'un test de dépistage d'une minute au résultat d'un test de dépistage validé de dix minutes pour le déficit cognitif léger (DCL) pour identifier les patients atteints d'un déficit cognitif (DC), chez des individus âgés de 65 ans ou plus qui présentent deux facteurs de risque vasculaires (FRV) ou plus. Méthodes : Des patients $(n=1523)$ âgés de 65 ans ou plus, sans symptôme de DC établi ou de démence mais qui présentaient deux FRV ou plus, ont participé à cette étude effectuée dans le contexte de soins de première ligne au Canada. Les données initiales étaient recueillies et l'Animal Fluency Test (AF) d'une minute et le Montreal Cognitive Assessment test (MoCA) de dix minutes étaient ensuite administrés. Les médecins $(\mathrm{n}=122)$ complétaient le cahier d'observation pendant l'entrevue avec le patient et rapportaient leur impression diagnostique. La sensibilité du test AF, sa spécificité et son exactitude pour prédire un MoCa positif ont été évaluées. Résultats : L'âge moyen des sujets était de 79,7 ans, 55\% étaient des femmes, $97,6 \%$ étaient des caucasiens et $75 \%$ avaient 12 ans ou moins de scolarité. Le test AF et le test MoCA ont détecté une DC chez $52 \%$ et $56 \%$ des sujets étudiés respectivement. La sensibilité du test AF, la spécificité et l'exactitude à prédire un test MoCA positif étaient toutes deux de 67\%. Selon l'impression diagnostique du médecin il s'agissait d'une DCL chez 37\% des patients et d'une démence chez 6\%. Conclusion : Dans une population âgée présentant au moins deux FRV, l'AF peut être utile pour détecter les symptômes d'un DC ou d'une démence jusque là non reconnus. Le dépistage d'un DC chez cette population à haut risque est justifié pour aider les médecins à identifier le début d'un DC. Le peu de temps requis pour compléter l'AF facilite son intégration en pratique clinique.

Can. J. Neurol. Sci. 2009; 36: 599-604

Dementia rates increase with age ${ }^{1}$ an analysis of 22 studies reported a doubling of dementia prevalence rates every 5.1 years until the age of $95 .^{2}$ The yearly incidence of dementia in Canada, among individuals aged 65 years and older, is estimated at 19.1/1000 for men and 21.8/1000 for women. ${ }^{3}$ The prevalence of dementia is expected to increase over the next decades, as a result of the aging population. ${ }^{4,5}$

Vascular events have been implicated in the pathogenesis of cognitive impairment (CI). ${ }^{6}$ Risk factors for CI leading to dementia include vascular disease. ${ }^{7}$ hvpertension. ${ }^{8}$ diabetes, ${ }^{8,9}$
From McMaster University (RS, LT), Hamilton; University of Ottawa (WD), Ottawa, ON; Université de Montréal (FM), Montréal; Centre Régional Ambulatoire de Laval (AT), Laval; BioMedCom Consultants Inc. (PM), Dorval; Pfizer Canada Inc. (PAL), Kirkland; Employed by Pfizer Canada Inc. (BL), Kirkland, QC; University of Calgary (RW), Calgary, AB, Canada.

Received November 6, 2008. Final Revisions Submitted March 31, 2009. Correspondence to: Pierre-Alexandre Landry, Pfizer Canada Inc., 17300 TransCanada Highway, Kirkland, Quebec, H9J 2M5, Canada. 
hyperlipidemia, ${ }^{10}$ and stroke. ${ }^{11}$ Among the elderly, the prevalence of vascular risk factors for dementia is high. ${ }^{12,13}$ As many as 86 percent of elderly psychiatric outpatients without a current diagnosis of dementia have at least one risk factor - the most common being hypertension (49\% of patients), heart disease (31\%), hypercholesterolemia (26\%), and diabetes mellitus $(23 \%) .{ }^{12}$ Because the rates of vascular events such as heart disease and stroke are known to increase with age, the prevalence of dementia is expected to rise even further. ${ }^{4,5}$

The early screening and identification of elderly subjects at high risk for dementia may improve the overall management of risk factors for vascular disease. ${ }^{14}$ Treating hypertension, diabetes, hyperlipidemia, and the cessation of smoking among high risk individuals may delay the onset or decrease the incidence of dementia. ${ }^{11,15,16}$ By delaying the onset of dementia, important gains can be made in terms of alleviation of the disease burden. ${ }^{17,18}$ It is estimated that delaying the onset of dementing illness by five years could reduce the prevalence of dementia by 50 percent. ${ }^{19}$ Furthermore, the early identification of patients with mild CI (MCI) may lead to interventions in the early stages of the disease that may slow the progression of cognitive decline..$^{20}$

Mild cognitive impairment is defined as memory impairment without associated functional impairment; MCI is considered a high risk state for progression to dementia. ${ }^{21}$ American Academy of Neurology guidelines recommend screening for MCI in high risk populations. ${ }^{22}$ The routine screening of individuals at risk for MCI has been shown to increase rates of dementia diagnosis. ${ }^{23}$

A clinical diagnosis of dementia requires a structured clinical approach consisting of patient history, physical assessment, and psychometric or cognitive testing. ${ }^{24}$ Full clinical and psychometric testing, however, is time consuming and difficult to incorporate into routine clinical practice. Screening instruments have been developed to assist physicians in the early detection of cognitive decline in high risk subpopulations. The Montreal Cognitive Assessment (MoCA) (a published cognitive screening tool, validated in English and French) assists primary care physicians in the detection of $\mathrm{MCI}$; with an average administration time of ten minutes, the test has shown high sensitivity and specificity for detecting MCI in patients that score within the normal range on the Mini Mental State Examination (MMSE). ${ }^{25}$ Although the time required to administer the MoCA is less than full clinical and psychometric testing, even the ten minutes required may limit its use in routine clinical practice. The animal fluency (AF) test provides an assessment of semantic fluency by asking patients to name as many animals as they can in one minute. Deficits in these cognitive domains are thought to be associated with damage to the temporal and parietal lobes as well as frontal-subcortical circuits. ${ }^{26}$ Animal fluency has been shown to distinguish patients with dementia from normal controls. ${ }^{26}$ Semantic fluency has been shown to be reduced in patients with MCI compared to cognitively intact controls. ${ }^{27}$

Canadian consensus guidelines recommend that physicians maintain a high index of suspicion for dementia and follow-up on their concerns when dealing with elderly patients that demonstrate memory loss and functional decline. ${ }^{24}$ It is recommended that physicians perform a comprehensive assessment for elderly patients with symptoms of CI, cognitive complaints or concerns. In real world clinical practice, proceeding from suspicion to diagnosis of dementia is often a lengthy process that does not occur at early stages when it would be the most helpful. As such, a care gap exists with the prevalence of undiagnosed dementia in its early stages remaining high; in a recent study, as few as $19 \%$ of patients with confirmed dementia had documentation of the illness on their medical record. ${ }^{28}$

The objective of this study was to evaluate - in the setting of routine primary care practice - the performance of a one-minute screening test measured against a validated ten-minute screening test for MCI in detecting CI in patients aged $\geq 65$ years who had two or more vascular risk factors (VRF).

\section{MeTHODS}

\section{Study Design}

DECIDE was an evaluation of a screening tool for MCI conducted at primary care settings across Canadian provinces (from September, 2005 to March, 2006). The study adhered to Good Clinical Practice and International Conference on Harmonization Guidelines, and the protocol was approved by appropriate independent research ethics committees (Institutional Review Board Services, Aurora, Ontario, and the University of Calgary Office of Medical Bioethics).

Primary care physicians $(n=122)$ who were familiar with the diagnosis and treatment of dementia and who had a patient population sufficiently at risk for dementia were enrolled from all provinces except Prince Edward Island. The number of physicians selected from each province was proportional to the share of the provincial elderly population aged 65 years and older, and ranged from 41 physicians in Ontario to 2 in New Brunswick. The annual prescription volume of anti-dementia medication in combination with cardiovascular or cholesterol lowering medications was used to determine if the physicians treated an adequate at-risk population allowing enrolment of sufficient patients within the study time frame. Duration of patient enrollment and completion of the study was estimated at approximately four months.

\section{Subjects}

To participate in the study, the patients $(n=1523)$ had to be at least 65 years-of-age, provide written consent, and have two or more of the following risk factors: type 2 diabetes; hypertension; hypercholesterolemia; cigarette smoking (current or past); obesity (BMI $\geq 30$ ); coronary artery disease (myocardial infarction or angina or coronary artery bypass graft); cerebrovascular disease (stroke or transient ischemic attack where deficits did not preclude administration of the AF test and MoCA); atrial fibrillation; congestive heart failure; or peripheral vascular disease. Patients were enrolled as they visited their primary care physicians for a routine visit.

Study exclusion criteria included: a diagnosis of dementia or documented CI prior to the study visit; evidence of current clinically significant depression or current alcohol abuse as defined by DSM-IV-TR criteria; enrollment in the present study on a previous occasion; and concurrent participation in a study with an experimental drug or a cholinesterase inhibitor. Patients 
who had any condition which, in the physician's judgment, might decrease the chance of obtaining satisfactory data to achieve the objectives of the study, were unable or unlikely to understand the nature, scope and possible consequences of the study, showed evidence of an uncooperative attitude, or were not sufficiently proficient in English or French to be assessed by the study instruments were also excluded from the study.

A stratified approach to patient enrollment was undertaken to optimize the balance between obtaining patient enrollment across all age groups and obtaining sufficient data on detected cases of CI. Each physician was asked to enroll three patients aged 65-74 years, seven patients aged 75-84 years, and five patients aged 85 years or older. Physicians continued their normal prescribing practices throughout the study.

\section{Data Collection}

During a single study visit for each patient, baseline data was collected (two-page clinical data form with: inclusion/exclusion criteria; socio-demographics; vascular risk factors and their levels of control; current and past medication use; family history; information regarding memory changes; current clinical impression and intended management approach). Primary care physicians completed case report forms during the patient interviews. They participated in a training session detailing the administration of the AF test and MoCA. The tests were administered by physicians and completed for each patient. Scores of 25 or less on the 30 point MoCA were considered indicative of $\mathrm{CI},{ }^{25}$ whereas the threshold for $\mathrm{CI}$ on the $\mathrm{AF}$ test was a score below $15 .^{26}$

\section{Data Analyses}

We used descriptive statistics to summarize the demographic and clinical characteristics of the sample expressed as mean for continuous variables and count (percent) for categorical variables. The purpose of the analysis was to compare the effectiveness of AF in detecting $\mathrm{CI}$ with the MoCA. As such, positive detection results with the AF test were considered true positive when they concurred with corresponding positive MoCA results and positive AF test results were considered false positive when the corresponding MoCA result was negative.

In the primary analysis, the AF positive predictive value was calculated as: (number of true positive AF test results)/(all positive AF test results), and the AF negative predictive value was determined by: (number of true negative AF test results)/(all negative $\mathrm{AF}$ test results). Compared to MoCA, AF test sensitivity was calculated as: (number of true positive AF test results)/(number of positive MoCA results), specificity was calculated using: (number of true negative AF test results)/(number of negative MoCA results), and accuracy was determined by the formula: (number of true positive AF test results + number of true negative $\mathrm{AF}$ test results)/(all positive $\mathrm{AF}$ test results + all negative AF test results). The discriminating power of the AF test relative to the threshold for CI (i.e., fewer than 15 animals named) was evaluated using Receiving Operator Characteristic (ROC) analysis, which is a technique that illustrates the trade-offs between the true positive rate (sensitivity) and the false positive rate (1-specificity) for every possible cutoff. The global index of screening accuracy is measured by the area under the ROC curve (AUC), where an
AUC of 1 represents a perfect screening test, and 0.5 corresponds to random assignment. ${ }^{29}$ All analyses were performed using SAS 9.2 (Cary, NC).

\section{RESULTS}

Patient characteristics are listed in Table 1. The mean age of the study population was 79.7 years [stratified into three groups: 65-74 years $(\mathrm{n}=363), 75-84$ years $(\mathrm{n}=704), 85$ years and older $(\mathrm{n}=443)$ ]. Fifty-five percent were female, Caucasians represented 97.6 percent of subjects, and 75 percent had less than 12 years of education. The most common cardiovascular disease (CV) risk factors were: high blood pressure (81\%); elevated cholesterol (58\%); coronary artery disease (37\%); and diabetes (33\%). The presence of multiple CV risk factors was common; 63 percent of subjects had three or more $\mathrm{CV}$ risk factors. The prevalence of concomitant drug treatments for CV risk factors was high among the study population with 87 percent of patients taking antihypertensive medications, 65 percent taking aspirin, 55 percent taking statins, and 27 percent taking anti-diabetic medications. Twenty-five percent of patients were taking sedatives or hypnotics.

Table 1: Patient characteristics at baseline

\begin{tabular}{lc}
\hline Patient characteristic & $\mathbf{1 5 1 2}$ \\
Subjects (n) & $24 / 47 / 29$ \\
65-74 yrs/75-84 yrs/85+ years (\%) & $45: 55$ \\
Sex, M:F (\%) & 97.6 \\
Caucasian (\%) & 75 \\
$\leq 12$ years education (\%) & 17 \\
Taken university courses (\%) & \\
Prevalence of individual vascular risk factors (\%) & 81 \\
High blood pressure & 58 \\
Elevated cholesterol & 37 \\
Coronary artery disease & 33 \\
Diabetes & 26 \\
Past smoker & 7 \\
Current smoker & 19 \\
Elevated BMI & 14 \\
Cerebrovascular disease & 13 \\
Atrial fibrillation & 11 \\
Peripheral vascular disease & 9 \\
Congestive heart failure & $37 / 34 / 19 / 10$ \\
2/3/4/5+ vascular risk factors $(\%)$ & \\
Family history of dementia or depression (\%) & 10 \\
Alzheimer's disease & 5 \\
Dementia & 7 \\
Depression & \\
\hline
\end{tabular}


Table 2: Overall effectiveness of the animal fluency test relative to the Montreal Cognitive Assessment in detecting cognitive impairment

\begin{tabular}{lcc}
\hline Outcome measure & $\begin{array}{c}\text { AF } \\
(\mathbf{n = 1 5 0 7 )}\end{array}$ & $\begin{array}{c}\text { MoCA } \\
(\mathbf{n = 1 5 0 7})\end{array}$ \\
Positive for cognitive impairment & 787 & 843 \\
Negative for cognitive impairment & 720 & 664 \\
Prevalence (\%) & 52 & 56 \\
& AF versus MoCA \\
Sensitivity n (\%) & $567 / 843(67)$ \\
Specificity n (\%) & $444 / 664(67)$ \\
Accuracy n (\%) & $1011 / 1507(67)$ \\
Positive predictive value n (\%) & $567 / 787(72)$ \\
Negative predictive value n (\%) & $444 / 720(62)$ \\
\hline
\end{tabular}

AF: Animal Fluency; MoCA: Montreal Cognitive Assessment defined as MCI (37\%) or dementia (6\%), versus 52 percent by the AF test and 56 percent by the MoCA.

\section{DISCUSSION}

Administered by physicians in primary care practice, the oneminute AF test is promising in detecting different levels of CI. When administered by physicians to patients without recognized $\mathrm{CI}$ or dementia, the AF test identified CI in 52 percent of patients compared to 56 percent by MoCA. Physician impression after administration of the $\mathrm{AF}$ and MoCA tests was that 43 percent of patients were cognitively impaired. Compared to MoCA, AF test accuracy in this population was 67 percent. The comparison of the sensitivity and specificity as a function of different cutoff points supported that a threshold of 15 animals named provided a good balance between true positive (sensitivity) and false positive (specificity) of the animal verbal fluency test for the screening of cognitive impairment.

A scatter plot of AF test scores versus MoCA scores demonstrated a trend towards a linear relationship between the number of correct answers in the AF test and the MoCA score. The few outliers with false negative and false positive test results tended to cluster near the AF test and MoCA thresholds for CI.

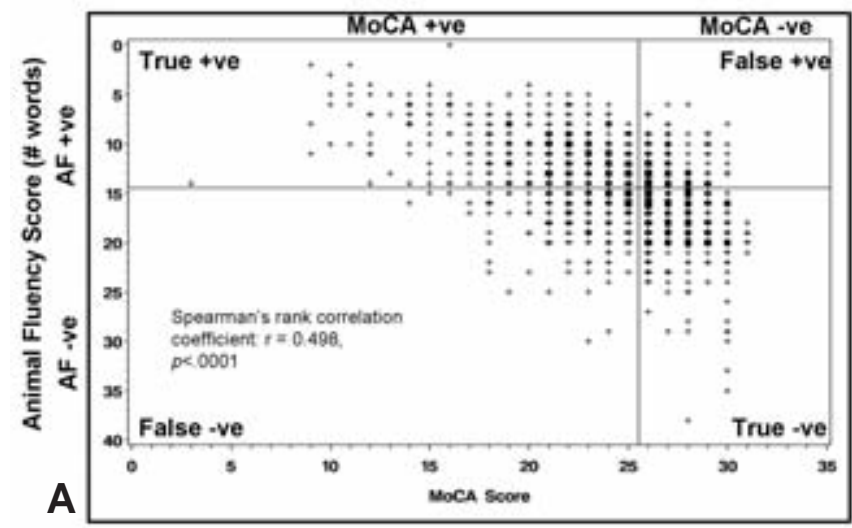

animals named, with relatively limited increase of the true positives (<14: specificity $58 \%$, sensitivity $76 \%$; <16: specificity $76 \%$, sensitivity $57 \%$ ).

Plotting the data by patient score on both the AF test and the MoCA, Figure A shows the results in the quadrant labelled 'true positive' for those patients scoring positive for CI on both tests, and in the quadrant labeled 'true negative' for those scoring negative for CI on both tests. The scatter plot of results indicates that as the MoCA score increases, so does the number of correct answers in the AF test. The association between AF and MoCA scores demonstrates a statistically significant relationship between the two measures, with most false negative and false positive results localized near the MoCA and AF published thresholds for $\mathrm{CI}^{26}$ (Spearman's rank correlation coefficient: $\rho=$ $0.498, p<.0001)$. Figure B portrays the proportion of patients screened with the AF and MoCA tests who scored positive for CI, alongside the proportion of patients evaluated by the physician as being cognitively impaired. According to physician diagnostic impression, after the sequential administration of AF and MoCA tests, 43 percent of patients had symptoms of CI

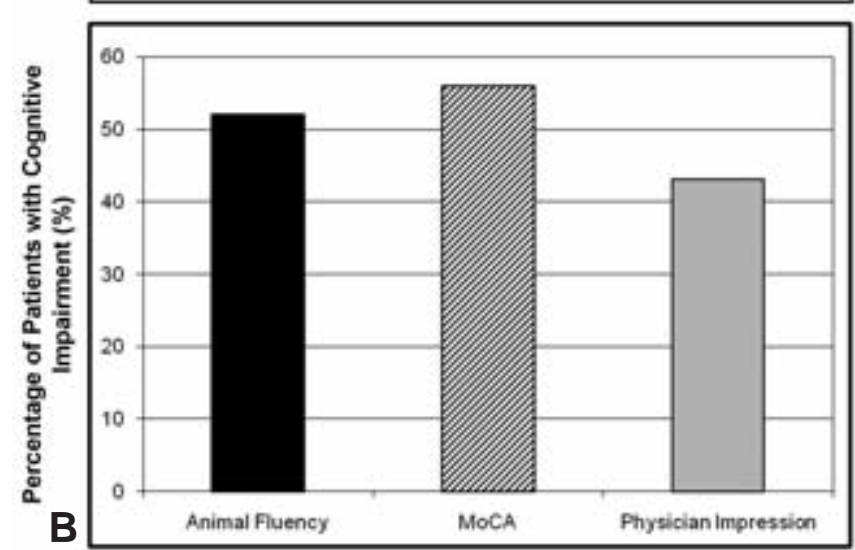

Figure: A. Scatter plot of animal fluency $(A F)$ versus Montreal Cognitive Assessment (MoCA) results in detecting cognitive impairment. $B$. Percentage of patients identified as having cognitive impairment using the AF test, the MOCA, or by physician diagnostic impression. 
The present study assessed the performance of a CI screening instrument in the context of a busy primary care practice. Physicians have identified limited available time as a barrier to dementia diagnosis. ${ }^{31} \mathrm{~A}$ brief screening instrument that serves to identify patients at-risk for CI or dementia and for whom further assessment is warranted would permit physicians to commit resources efficiently. The AF test is quick to administer-an important characteristic for the routine implementation of CI screening in the primary care setting.

The prevalence of dementia is expected to rise substantially in the coming years, ${ }^{5}$ as will its impact on patients and society. Dementia is under-diagnosed; more than 50 percent of patients with dementia have not been diagnosed. ${ }^{20}$ In the present study, 43 percent of patients were eventually judged by their physicians as having CI and, in some cases, dementia even though a diagnosis of CI or dementia were study exclusion criteria. It is important to identify CI before it progresses to dementia. In cases where dementia has developed, initiating treatment for dementia early in the disease process can be beneficial. ${ }^{20}$ In those with early stage disease, cholinesterase inhibitors have been shown to consistently slow the rate of decline in cognitive and global clinical change scores, as well as functional and behavioural measures that can contribute to a reduction of patient and caregiver burden. ${ }^{20}$ In the future, when disease modifying agents become available, it will be even more important to have practical useful cognitive screening tests.

Cardiovascular risk factors are positively associated with cognitive decline. ${ }^{6,8}$ Hypertension, heart disease, hypercholesterolemia, and other vascular risk factors are common among the elderly. ${ }^{12}$ Significant gains can be made by the timely identification of individuals at-risk and working towards reducing the modifiable risk factors. Among the more than 2800 subjects taking part in the Systolic Hypertension in Europe Study (SYST-EUR), long-term antihypertensive therapy led to a 55 percent reduction in dementia over a period of 3.9 years. ${ }^{16}$ Although an infarct in a particularly sensitive brain region can produce a dramatic effect on cognitive function, cognitive decline is often the result of multiple brain lesions accumulated over time..$^{5,8}$ By identifying elderly patients with $\mathrm{CV}$ risk factors early, a simple screening test can help determine a course of action to reduce the progression rate to dementia. ${ }^{22}$ Delaying the onset of dementia would substantially decrease the prevalence of the disease, and reduce patient, caregiver and societal burdens. ${ }^{17,19}$

Other tests for CI have been described elsewhere. The MMSE - a test which has high specificity and sensitivity for detecting dementia in outpatients older than 65 years $^{32}-$ is the most commonly used test to screen for memory problems and for diagnosing dementia. ${ }^{33}$ However, the MMSE has a ceiling effect, demonstrating a limitation of the test in identifying early dementia. ${ }^{34}$ The MoCA has been shown to have higher sensitivity in detecting MCI compared to the MMSE. ${ }^{25}$ The present study showed that the brief AF test identified only four percent fewer individuals with $\mathrm{CI}$ than the MoCA.

\section{Study Limitations}

Although the MoCA was used here as a comparator, the true gold standard for the detection of CI is full clinical and psychometric testing. However, given the time requirement to conduct such an evaluation, it was deemed unrealistic to include this for the study or within the context of a busy clinical practice. The AF test is a brief instrument that assesses two domains of cognitive functioning whereas MoCA evaluates several cognitive domains. The simplicity of the AF test allows for brevity, however, some specificity is lost as evidenced by the 67 percent accuracy of the test when compared to another screening instrument-MoCA.

The one minute AF test is a short screening tool that may assist in screening for CI in the primary care setting in patients who are 65 years or older and have two or more vascular risk factors. By identifying patients at risk for progression to dementia, further testing and appropriate measures to counter the progression of the disease can be undertaken.

\section{ACKNOWLedgMents}

The authors thank Annie Petrie at Clinforma Data Systems and Management for project management, data handling and analysis, Dr. Gary Foster, McMaster University, for assisting with the core statistical analyses, and the DECIDE study investigators. This article was prepared with the assistance of BioMedCom Consultants inc, Montreal, Canada. The study was funded by Pfizer Canada Inc.

\section{REFERENCES}

1. Brayne C, Gao L, Dewey M, Matthews FE. Dementia before death in ageing societies--the promise of prevention and the reality. PLoS Med. 2006;3(10):e397.

2. Jorm AF, Korten AE, Henderson AS. The prevalence of dementia: a quantitative integration of the literature. Acta Psychiatr Scand. 1987;76(5):465-79.

3. The incidence of dementia in Canada. The Canadian Study of Health and Aging Working Group. Neurology. 2000;55(1): 66-73.

4. Roman GC. Stroke, cognitive decline and vascular dementia: the silent epidemic of the 21 st century. Neuroepidemiology. 2003;22(3):161-4.

5. Grossman H, Bergmann C, Parker S. Dementia: a brief review. Mt Sinai J Med. 2006;73(7):985-92.

6. Martinez-Vila E, Murie-Fernandez M, Gallego Perez-Larraya J, Irimia P. Neuroprotection in vascular dementia. Cerebrovasc Dis. 2006;21 Suppl 2:106-17.

7. Brayne C, Gill C, Huppert FA, Barkley C, Gehlhaar E, Girling DM, et al. Vascular risks and incident dementia: results from a cohort study of the very old. Dement Geriatr Cogn Disord. 1998; 9(3): $175-80$.

8. Knopman D, Boland LL, Mosley T, Howard G, Liao D, Szklo M, et al. Cardiovascular risk factors and cognitive decline in middleaged adults. Neurology. 2001;56(1):42-8.

9. Gregg EW, Yaffe K, Cauley JA, Rolka DB, Blackwell TL, Narayan $\mathrm{KM}$, et al. Is diabetes associated with cognitive impairment and cognitive decline among older women? Study of Osteoporotic Fractures Research Group. Arch Intern Med. 2000;160(2): 174-80.

10. Trinh N-H, Yaffe K. Alzheimer's disease: incidence research and treatment. Behavioral health trends. Drug Benefit Trends. 2004; 16(Suppl 5):27S-35S.

11. Sachdev PS, Brodaty H, Looi JC. Vascular dementia: diagnosis, management and possible prevention. Med J Aust. 1999;170(2): 81-5.

12. Stella F, Banzato CE, Gasparetto Se EV, Scudeler JL, Pacheco JL, Kajita RT. Risk factors for vascular dementia in elderly psychiatric outpatients with preserved cognitive functions. J Neurol Sci. 2007;257(1-2):247-9.

13. Wilson PW, Kannel WB. Obesity, diabetes, and risk of cardiovascular disease in the elderly. Am J Geriatr Cardiol. 2002; 11(2):119-23,125. 
14. Roman GC. Vascular dementia; changing the paradigm. Curr Opin Psychiatry. 2003;16(6):635-41.

15. Alagiakrishnan $\mathrm{K}, \mathrm{McC}$ racken $\mathrm{P}$, Feldman $\mathrm{H}$. Treating vascular risk factors and maintaining vascular health: is this the way towards successful cognitive ageing and preventing cognitive decline? Postgrad Med J. 2006;82(964):101-5.

16. Forette F, Seux ML, Staessen JA, Thijs L, Babarskiene MR, Babeanu S, et al. The prevention of dementia with antihypertensive treatment: new evidence from the Systolic Hypertension in Europe (Syst-Eur) study. Arch Intern Med. 2002;162(18):2046-52.

17. Van Gool WA. Can we prevent, delay, or shorten the course of dementia? PLoS Med. 2006;3(10):e430.

18. Brookmeyer R, Johnson E, Ziegler-Graham K, Arrighi HM. Forecasting the global burden of Alzheimer's disease. Alzheimers Dement. 2007;3(3):186-91.

19. Thal LJ, Carta A, Doody R, Leber P, Mohs R, Schneider L, et al. Prevention protocols for Alzheimer disease. Position paper from the International Working Group on Harmonization of Dementia Drug Guidelines. Alzheimer Dis Assoc Disord. 1997;11 Suppl 3: 46-9.

20. Boustani M, Peterson B, Hanson L, Harris R, Lohr KN. Screening for dementia in primary care: a summary of the evidence for the U.S. Preventive Services Task Force. Ann Intern Med. 2003;138(11):927-37.

21. Forti P, Maioli F, Pisacane N, Rietti E, Montesi F, Ravaglia G. Atrial fibrillation and risk of dementia in non-demented elderly subjects with and without mild cognitive impairment. Neurol Res. 2006;28(6):625-9.

22. Petersen RC, Stevens JC, Ganguli M, Tangalos EG, Cummings JL, DeKosky ST. Practice parameter: early detection of dementia: mild cognitive impairment (an evidence-based review). Report of the Quality Standards Subcommittee of the American Academy of Neurology. Neurology. 2001;56(9):1133-42.

23. Borson S, Scanlan J, Hummel J, Gibbs K, Lessig M, Zuhr E. Implementing routine cognitive screening of older adults in primary care: process and impact on physician behavior. J Gen Intern Med. 2007;22(6):811-7.

24. Patterson CJ, Gauthier S, Bergman H, Cohen CA, Feightner JW, Feldman $\mathrm{H}$, et al. The recognition, assessment and management of dementing disorders: conclusions from the Canadian Consensus Conference on Dementia. CMAJ. 1999;160 Suppl 12:S1-15.
25. Nasreddine ZS, Phillips NA, Bedirian V, Charbonneau S, Whitehead V, Collin I, et al. The Montreal Cognitive Assessment, MoCA: a brief screening tool for mild cognitive impairment. J Am Geriatr Soc. 2005;53(4):695-9.

26. Canning SJ, Leach L, Stuss D, Ngo L, Black SE. Diagnostic utility of abbreviated fluency measures in Alzheimer disease and vascular dementia. Neurology. 2004:62(4):556-62.

27. Nutter-Upham KE, Saykin AJ, Rabin LA, Roth RM, Wishart HA, Pare N, et al. Verbal fluency performance in amnestic MCI and older adults with cognitive complaints. Arch Clin Neuropsychol. 2008;23(3):229-41.

28. Boustani M, Callahan CM, Unverzagt FW, Austrom MG, Perkins AJ, Fultz BA, et al. Implementing a screening and diagnosis program for dementia in primary care. J Gen Intern Med. 2005; 20(7):572-7.

29. Fawcett T. An introduction to ROC analysis. Pattern Recognition Letters. 2006;27:861-74.

30. Millis S. Neuropsychology of malingering casebook: Chapter 3. London: Taylor \& Francis; 2008.

31. Boise L, Camicioli R, Morgan DL, Rose JH, Congleton L. Diagnosing dementia: perspectives of primary care physicians. Gerontologist. 1999;39(4):457-64

32. Harvan JR, Cotter V. An evaluation of dementia screening in the primary care setting. J Am Acad Nurse Pract. 2006;18(8): $351-60$.

33. Alzheimer's Society. The Mini Mental State Examination (MMSE): a guide for people with dementia and their carers. Alzheimer's Society Quality Research in Dementia information sheet. 2002. (Accessed 18 Jan 2007) http://www.alzheimers.org.uk/ How_is_dementia_diagnosed/Diagnosis_process/info_mmse.ht m. [cited 2007 Jan 18].

34. Ihl R, Frolich L, Dierks T, Martin EM, Maurer K. Differential validity of psychometric tests in dementia of the Alzheimer type. Psychiatry Res. 1992;44(2):93-106. 